\title{
Cannce?
}

\section{CannX: The International Medical Cannabis Conference Lisbon, Portugal, February 12-13, 2020}

\section{Abstracts}

\section{Scientific Committee}

\section{Prof. Lumir Hanus}

Chief Scientist, Lumir Lab, Associate Professor, Palacký University, Jerusalem Biotechnology Park, Hebrew University, Ein Kerem Campus, Senior Fellow, IEHP, Thomas Jefferson University, Philadelphia, USA, Professor Chaver and Research Fellow, Hebrew University (Ret.) Institute for Drug Research, School of Pharmacy, Faculty of Medicine, Hebrew University, Israel

\section{Catherine Jacobson}

VP, Regulatory \& Medical Affairs, Tilray, USA

\section{Prof. Renato Malcher-Lopes}

Department of Physiological Sciences, Institute of Biological Sciences, University of Brasilia, Brazil

\section{Prof. Raphael Mechoulam}

Institute for Drug Research, The Hebrew University of Jerusalem, Israel

\section{Dr. David (Dedi) Meiri}

Head of Scientific Advisory Committee Assistant Professor, Heads the "Laboratory of Cancer Biology and Cannabinoid Research" Technion, Institute of Technology, Israel

\section{Dr. Orit Stolar}

Pediatric Neurologist, Assaf Harofeh Medical Center, Israel

\section{Dr. Yuval (Tuby) Zolotov}

Network and Partnership Coordinator, International Association for Cannabinoid Medicine, Israel

\footnotetext{
Karger $\stackrel{2}{=}$

Basel · Freiburg · Paris · London · New York · Chennai · New Delhi ·

Bangkok · Beijing · Shanghai · Tokyo · Kuala Lumpur · Singapore · Sydney
} 


\section{Science: Innovations in Science and Medicine}

\section{1}

\section{Evaluation of Anticancer Properties of Different Extracts of Cannabis Sativa Cultivated in Pakistan Against Some Common Cancers: A Study Protocol G. Afshan ${ }^{1}$, S. Khan ${ }^{2}$, R. Khan ${ }^{1}$, S. Siddiqi ${ }^{1}$ \\ ${ }^{1}$ The Aga Khan University, Anaesthesiology, Karachi, Pakistan; \\ ${ }^{2}$ The Aga Khan University, BBS, Karachi, Pakistan}

Cancer is the second leading cause of death globally and is responsible for an estimated 9.6 million deaths in 2018. Reported cases of cancer in both genders are 18.78 million in 2018 with approximately $70 \%$ of deaths occurring in low- and middle-income countries, including Pakistan. Breast cancer is the highest reported cancer in Pakistan while prostate cancer constitutes $6.7 \%$ of the total cancer burden. Anti-cancer activity of selected plant-derived cannabinoids has been studied in different cancer cell lines showing positive results by some investigators but there is no such report available from Pakistan and the therapeutic potential of Pakistani varieties of Cannabis Sativa remains unexplored. We infer that the variant of C. sativa cultivated in Pakistan is efficacious against human breast cancer and prostate cancer cell lines. This study is planned to evaluate the anticancer properties of different extracts (Flowers, leaves, stem, root, seeds and whole plant extracts) of Cannabis Sativa cultivated in Pakistan against human breast cancer cell lines (MCF-7 and MDA-MB-231) and prostate cancer cell lines (PC3 and LNCaP). All plant material will be dry and powdered in pharmacology laboratory. Each plant material will be soaked in aqueous methanol (30:70) for three days at room temperature and 1st, 2nd, and 3rd filtrates will be obtained using a muslin cloth and Whatman filter paper. The filtrates will be combined together and solvent will be removed using a rotary-evaporator under reduced pressure, to yield/obtained respective Cannabis sativa thick extract. An intramural grant has been procured for this study. Results will be shared during the research presentation.

\section{2 \\ Effects of Delta-9-Tetrahydrocannabinol in BeWo and HTR-8 Placental Cells}

$\underline{\text { P. Alves }}^{1}$, C. Amaral ${ }^{2}$, J. Maia ${ }^{2}$, N. Teixeira ${ }^{2}$, G. Correia-Da-Silva ${ }^{2}$

${ }^{1}$ Faculty of Pharmacy, University of Porto, Biological Sciences, Porto, Portugal; ${ }^{2}$ UCIBIO, REQUIMTE, Biological Sciences, Porto, Portugal

Delta-9-tetrahydrocannabinol (THC) is the main phytocannabinoid in "Cannabis sativa". Cannabis use by pregnant women to relieve morning sickness has been increasing, as reported by various epidemiological studies. Diverse processes are involved in the remodelling of trophoblast cells, such as proliferation, differentiation, apoptosis and invasion, which are crucial for placental development. The consumption of cannabis during pregnancy is of much concern since it may disrupt the endocannabinoid system (ECS) which modulates several biological functions, including reproduction. Our group demonstrated that THC has an impact on trophoblast turnover, through induction of apoptosis, as well as on the ECS homeostasis. Considering the relevance of cannabinoid signalling in reproductive tissues, women of child-bearing age may be a particularly sensitive group to cannabinoids exposure. Thus, it is important to understand the consequences of this exposure on reproductive health. The aim of this work was to study the effects of THC on trophoblast cells, using BeWo and HTR-8 (ATCC, USA) cell lines, well-accepted models of cytotrophoblasts ("stem cells") and extravillous/invasive trophoblasts, respectively. It was observed that THC induces a dose-dependent decrease in cell viability although at lower concentrations in the case of HTR-8 cells. Results indicate that THC may affect trophoblast cells remodelling by apoptosis activation. Moreover, this cannabinoid affects the invasive properties of extravillous trophoblasts. Therefore, this work suggests that phytocannabinoids use during pregnancy may disrupt normal placental development and, consequently, impair pregnancy outcome. Funding: European Union (FEDER funds through COMPETE) and Fundação para a Ciência e Tecnologia (FCT) through project PTDC/DTP-FTO/5651/2014-POCI01-0145-FEDER-016562. The authors are grateful to FCT for Cristina Amaral Post-Doc grant (SFRH/BPD/98304/2013) and for João Maia PhD grant (BD/136105/2018).
C 2020 The Author(s)

Published by S. Karger AG, Basel

This article is licensed under the Creative Commons AttributionNonCommercial-NoDerivatives 4.0 International License (CC BYNC-ND) (http://www.karger.com/Services/OpenAccessLicense). Usage and distribution for commercial purposes as well as any distribution of modified material requires written permission. 


\section{3}

\section{Unveiling the Mechanisms Behind Cannabinoids as Potential Adjuvant Therapy for $\mathrm{ER}^{+}$Breast Cancer}

\section{Amaral, F. Trouille, C. Almeida, G. Correia-Da-Silva, N. Teixeira}

UCIBIO, REQUIMTE, Faculty of Pharmacy, University of Porto, Biochemistry Laboratory, Department of Biological Sciences, Porto, Portugal

Breast cancer is the main cause of cancer death in women worldwide. Estrogen receptor positive $\left(\mathrm{ER}^{+}\right)$breast cancer are around $75 \%$ of all breast cancer cases. Aromatase inhibitors (AIs) are the current first-line treatment for $\mathrm{ER}^{+}$breast cancer, as they prevent estrogen production and thus cancer progression [1]. Unfortunately, despite their therapeutic efficacy, resistance to AIs can arise after prolonged treatment, thus it is important to discover new targets or strategies to improve breast cancer treatment. The anti-cancer properties of cannabinoids have already been shown in several cancer cell lines and tumour types [2]. Moreover, we recently showed that cannabinoids have the ability to target and inhibit aromatase, the enzyme responsible for estrogen synthesis [3]. Considering this, in this work we studied the anti-cancer properties of the main endocannabinoid anandamide (AEA), as well as, $\Delta^{9}$-tetrahydrocannabiol (THC), on a human $\mathrm{ER}^{+}$breast cancer cell line that overexpresses aromatase (MCF-7aro). Results demonstrated that AEA and THC target aromatase and ERa, which is a therapeutic advantage, as these are key targets for ER+ breast cancer cell growth. Moreover, both cannabinoids impaired the growth of MCF-7aro cells. Thus, this work indicates that AEA and THC present promising anti-tumour properties in this type of cancer, which highlights these cannabinoids as potential and attractive adjunvant therapy for ER+ breast cancer. Acknowledgments: Fundação para a Ciência e Tecnologia (FCT) for Cristina Amaral Post-doc grant (SFRH/BPD/98304/2013; DL 57/2016 - Norma Transitória) and financial support (UID/Multi/04378/2019).

References

1 Augusto T, et al. (2018) Endocr Relat Cancer; 25(5):R283-R301.

2 Fonseca BM, et al. (2017) Rev Physiol Biochem Pharmacol; 173:63-88.

3 Almada M, et al. (2019) Biochim Biophys Acta Mol Cell Biol Lipids; 1864(12):158512.

\section{4}

\section{Use of Cannabis in People Living with HIV in Portugal}

F. De Aquiar ${ }^{1}$, P. Costa ${ }^{2}$, A. Silva ${ }^{3}$, F. Neves ${ }^{3}$, V. Duque 4 , C. Barbosa ${ }^{5}$

${ }^{1}$ Fundação Portuguesa A Comunidade Contra a SIDA, Administrative Council, Lisboa, Portugal; ${ }^{2}$ Fundação Portuguesa "A Comunidade Contra a SIDA" - FPCCSIDA, Fpccsida, Lisbon, Portugal; ${ }^{3}$ Centro Hospitalar do Porto, EPE - CHP, Chp, Oporto, Portugal; ${ }^{4}$ Centro Hospitalar e Universitário de Coimbra CHUC, Chuc, Coimbra, Portugal; ${ }^{5}$ Centro Direito Biomédico Universidade de Coimbra, Cdb, Coibra, Portugal

Despite advances in antiretroviral therapy for HIV infection, there is a growing need to control symptoms and long-term side effects throughout therapy. Noting the advantages of cannabis use reported in a set of common symptoms and complications of HIV infection, including loss of appetite, depression, neuropathy and difficulty sleeping, among others, we wanted to understand the benefits of using it over two years of cannabis oil above 15\% CBD content in 100 users in follow-up and follow-up at our institution. Data were collected through speeches at the consultation moments and answers to a questionnaire. Patients reported significant improvements in several symptoms: appetite (93\%), nausea $(88 \%)$, depression $(87 \%)$, anxiety (89\%) and sleep (96\%), muscle pain (94\%), nerve pain (78\%) and paraesthesia (88\%). Most users (78\%) assume improvements in overall well-being and quality of life from symptom improvement compared with a recent past.

\section{5}

\section{Cannabis Between Medical Uses and Recreational Uses}

\section{M.E. Ibrahim}

Faculty of Pharmacy, October 6 University, Pharmacognosy, Sixth of October City, Egypt

Introduction: There are a number of biologically active chemicals in Cannabis. The main psychoactive ingredients are called cannabinoids, and the primary cannabinoid produced is delta9-tetrahydrocannabinol (THC.). Cannabinoids are produced in the stalk, leaves, flowers, seeds and resin of marijuana plants. Cannabis can be smoked, vaporized, or eaten, among other forms of ingestion. THC is rapidly absorbed, and when inhaled, reaches the brain within minutes. However its oral absorption is lower owing to a significant reduction after passing though the liver. These chemicals bond to cannabinoid receptors on cells throughout the body, triggering or modulating different effects. Cannabis immediately affects attention, concentration, memory, learning and motor coordination, proportional to the dose, through the endocannabinoid system in our body. It is absolutely plausible that drugs that target endocannbinoid receptors, like THC (or derivatives), have the potential to produce beneficial medicinal effects. Objectives: Given the presence of cannabinoid receptors on nearly every organ system and with the growing understanding of the endocannabinoid system, and the identification of different types of receptors, there's the potential for targeting specific effects on specific organs. That could mean products that produce beneficial effects and minimize any adverse effects. Materials and Methods: By reviewing records of some patients with different types of pain after treatment with medical cannabis to know more about the medical effects of cannabis and its side effects. Results: Evidence for inhaled cannabis for pain is poor to provide good evidence-based guidance. Synthetic medical cannabis-derived products may improve neuropathic pain, chronic pain and cancer pain but perhaps not for other pain types. Dronabinol improves control of nausea/ vomiting post chemotherapy $\geq 40 \%$. Nabiximols likely improve multiple sclerosis spasticity $\geq 30 \%$. Patients' preference for cannabinoids exceeds cannabinoids effectiveness. Medical cannabinoids cause different adverse events in patients, from visual disturbance or hypotension to hallucination or paranoia. Conclusions: The use of psychoactive drugs like Cannabis is a health issue, particularly when used for medical purposes. Regrettably, there is a lack of 
high-quality data that shows Cannabis for most medical purposes is both safe and effective. However, clinical trials will hopefully become more common and more cannabis-based drugs can be more rigorously evaluated.

\section{6 \\ Classification of Medical Cannabis Strains for Pain Treatment by Zebrafish Larvae Model}

P. Pitashny ${ }^{1,2}$, R. Harari ${ }^{2}$, D. Goldberg ${ }^{1,2}$, K. Jackson ${ }^{1,2}$

${ }^{1}$ Tel Hai College, Nutrition and Food Sciences, K. Shmona, Israel;

${ }^{2}$ Migal, Human Nutrition and Health, K Shmona, Israel

The zebrafish (Danio rerio) had become a popular organism in neuroscience research for modeling brain diseases. This vertebrate shows external fertilization and rapid external development. The eggs and embryos are transparent, enabling neuronal imaging in vivo, and the mating of two adult fish, which can happen daily, supply a large clutch of offspring. The larvae small size contributes to an efficient, rapid, and cheap method to screen large numbers of chemicals trough behavioral assays and is considered the only suitable vertebrate model for high throughput drug or medicinal plants screening. Medicinal Cannabis (MC) become a complementary therapy for several health problems such as anxiety, pain, post-traumatic stress disorder, sleep disorders, convulsions, symptoms regarding neurodegenerative diseases (tremors in Parkinson's disease) and autism. Today many different cannabis strains differ in their chemical composition and, thus, the biological effect. There is a need for specific preclinical assays, which could determine the potential biological effect of each strain. Since the zebrafish larvae present the endocannabinoid system ECS in its whole showing the same neurotransmitters and receptors as mammals, this biological model is an appropriate tool for the detection and classification of different MC strains for different conditions. In the present work, we studied the effect of different MC strains with different concentrations of cannabinoids on pain. Six days-postfertilization zebrafish larvae in a 96-well plate were exposed to increasing doses of MC extracts of different strains or analgesic drugs (Buprenorphine). The larvae were then exposed to a painful stimulus (high temperature), which caused an increase in motor activity. The change in motor activity was measured using a Danio vision larva tracking system and expressed as a motion index. Analgesic drugs and specific cannabis strains lowered the motor activity to control levels. In conclusion, the zebrafish model could be an appropriate tool to identify effective MC strains for the treatment of pain and could be used for screening of different strains and further for clarifying the MC mechanism of action related to the analgesia.
The Rational and Clinical Use of Cannabinoids in Psychiatry: How It Works in Clinical Practice

M. Katzman ${ }^{1}$, T. Sternat ${ }^{2}$, I. Epstein ${ }^{1}$

${ }^{1}$ START Clinic for Mood and Anxiety Disorders, Psychiatry, Toronto, Canada; ${ }^{2}$ Adler Graduate Professional School,

Psychiatry, Toronto, Canada

There has been an exponential growth in our understanding in the literature that the endogenous endocannabinoid system is implicated across numerous vital physiological processes. Various components of cannabis are indicated in a number of medical conditions including: multiple sclerosis, childhood epilepsy, autoimmune conditions, and chronic pain syndromes. Research is oriented towards gaining a better understanding of the underlying neurobiological mechanisms in the central nervous system and brain and associated physiological connections of the endocannabinoid system, which may be implicated in some mental illnesses. However, in our current climate, the majority of physicians still remain hesitant to prescribe cannabinoids in the primary or adjunctive treatment of mood and anxiety disorders in their clinical practices. Recent literature has demonstrated or implicated abnormal levels of endogenous cannabinoids in depressive disorders, eating disorders, and schizophrenia. There is evidence and ongoing clinical trials that have demonstrated promising results for the treatment of post-traumatic stress disorder, ADHD, generalized and social anxiety disorders. However, to date there is a paucity of large studies, which are validated and evidence-based, with respect to patient selection criteria, suitable age-range for initiating treatment, and potential negative outcomes or risks associated with short and long-term use. More research is warranted in order to address safety concerns and contraindications related to the use of individual cannabinoids or combinations of cannabinoids, and the biological actions of terpenes that differ amongst various plant strains, especially in terms of their effects on the brain and soma. Concerns also centre on the impact of cognition in young individuals or the elderly. This symposium aims to review and propose a model for clinical use based on our current understanding of the neurobiology of the endocannabinoid system in the management of psychiatric illness, with a focus on potential therapeutic effects, risks and current guidelines. There is potential for a paradigm shift in therapeutic approaches in our management of psychiatric conditions. 


\section{8}

\section{Considerations for Clinical Trials with Children Evaluating Cannabinoids}

\author{
L. Kelly' 1 J. Alcorn², R. Huntsman ${ }^{3}$, T. Oberlander', \\ T. Lacaze-Masmonteil ${ }^{5}$, A. Palmer ${ }^{6}$, V. Tsang ${ }^{7}$, Y. Finkelstein ${ }^{8}$, \\ C. Moore-Hepburn' ${ }^{9}$ G. 'T Jong ${ }^{10}$
}

${ }^{1}$ University of Manitoba, Pediatrics and Child Health, Winnipeg, Canada; ${ }^{2}$ University of Saskatchewan, College of Pharmacy and Nutrition, Saskatoon, Canada; ${ }^{3}$ University of Saskatchewan, College of Medicine, Saskatoon, Canada; ${ }^{4}$ University of British Columbia, Pediatrics, Vancouver, Canada; ${ }^{5}$ University of Calgary, Pediatrics, Calgary, Canada; ${ }^{6} \mathrm{Ac} 2 \mathrm{orn}, \mathrm{Na}$, Toronto, Canada;

${ }^{7}$ KidsCan YPAG, Na, Vancouver, Canada; ${ }^{8}$ University of Toronto, Pediatrics, Toronto, Canada; ${ }^{9}$ Hospital for Sick Children, Na, Toronto, Canada; ${ }^{10}$ University of Manitoba, Pediatrics and Child Health, Winipeg, Canada

Half of Canadian paediatricians manage at least one child using cannabis products to address health concerns [1]. Currently there is considerable uncertainty regarding safe and effective dosing of cannabis in children. Over and above the usual challenges of conducting clinical research in children, cannabis presents additional challenges concerning paediatric pharmacokinetics, product quality, lack of evidence to inform dosing, social stigma, considerations for long term neurodevelopment, and a poorly understood endocannabinoid system. The Canadian Childhood Cannabinoid Clinical Trials (C4T) platform has been developed specifically to address these challenges and research gaps related to studying cannabis in children. C4T includes parents, doctors and scientists from all across Canada, representing 17 maternal child health research institutes. Rising interest in medical cannabis in patients with cancer, including children has been reported $[2,3]$. Whilst clinical trials are needed to provide the evidence-base, we must also provide timely answers to questions clinicians receive daily from parents on what products to use and how much is safe. Our overarching goal is to build and strengthen $\mathrm{C} 4 \mathrm{~T}$ in preparation for the successful design and conduct of future randomized controlled trials to evaluate the effectiveness of cannabis in children, starting with childhood cancers. Updates from C4T will be provided by our Scientific Director on our parent-resource hub, surveillance studies, observational studies on cannabis pharmacodynamics and pharmacokinetics in children with cancer and our open-label dosing study on a cannabis health product for symptom management in children with cancer. This session will also discuss practical considerations for operating cannabis clinical trials with children including ethical considerations, funding models and academic partnerships.

\section{References}

1 Grant CN, Belanger RE. Cannabis and Canada's children and youth. Paediatr Child Health 2017;22:98-102.

2 Kelly LE, Junker A. Considerations for clinical trials to study cannabinoids in Canadian children with neurologic disorders. CMAJ 2018;190: E1002-E3.

3 Hawley P, Gobbo M. Cannabis use in cancer: a survey of the current state at BC Cancer before recreational legalization in Canada. Curr Oncol 2019;26:e425-e32.
9

\section{Cannabis Use in Adolescent Chronic Daily Headache;} A Youth-Informed Tolerability Study

\author{
L. Kelly ${ }^{1}$, Z. Zaslawski ${ }^{1}$, J. Alcorn ${ }^{2}$, R. Huntsman ${ }^{3}$, T. Oberlander ${ }^{4}$, \\ E. Lewis ${ }^{5}$, A. Finley ${ }^{6}$ \\ ${ }^{1}$ University of Manitoba, Pediatrics and Child Health, Winnipeg, \\ Canada; ${ }^{2}$ University of Saskatchewan, College of Pharmacy and \\ Nutrition, Saskatoon, Canada; ${ }^{3}$ University of Saskatchewan, \\ College of Medicine, Saskatoon, Canada; ${ }^{4}$ University of British \\ Columbia, Pediatrics, Vancouver, Canada; ${ }^{5}$ Neurology Centre of \\ Toronto, Na, Toronto, Canada; ${ }^{6}$ Dalhousie University, Anesthesia, \\ Pain Management \& Perioperative Medicine, Halifax, Canada
}

Chronic daily headache $(\mathrm{CDH})$ is a common and debilitating disorder defined as headache occurring more than 15 days per month for longer than three months [1]. An epidemiological study of 9,000 school aged children reported $2.5 \%$ of children ( 7 years and above) and $15 \%$ of adolescents ( 15 years or older) experienced frequent headaches [2]. $\mathrm{CDH}$ are associated with severe morbidity including dizziness, nausea, vomiting, light sensitivities, reduced quality of life and facial numbness [3]. In Canada, adolescents with $\mathrm{CDH}$ are using cannabis products off-label to manage their symptoms, self-guided in the absence of evidence [4]. With the exception of epilepsy and chemotherapy induced nausea, limited studies have formally evaluated the risks and potential benefits of cannabinoids for therapeutic purposes in children. We propose a youthinformed Phase I open-label dose finding study to evaluate the safety and dosing of a CBD-enriched cannabis product in adolescents with primary $\mathrm{CDH}$. The main objective of this dose finding study is to determine if a CBD-enriched cannabis product is well tolerated in adolescents with $\mathrm{CDH}$. Our secondary objectives are to determine the relationship between dose escalation with steadystate trough plasma levels of cannabinoids and metabolites. We will engage with a young persons' advisory group to inform study design. Twenty adolescents, age $14-18$ years with primary $\mathrm{CDH}$ for more than 6 months that have failed other treatment option, will be recruited from three chronic pain clinics in Canada. They will receive scheduled dose increases and we will measure plasma concentrations of cannabinoids and metabolites. We hypothesize that a CBD-enriched product will be well tolerated up to a maximum dose of $10 \mathrm{mg} / \mathrm{kg} /$ day. The successful outcomes from this research project have the potential to create meaningful impact for the lives of Canadian adolescents given the high prevalence of chronic daily headaches, severe morbidity, and current lack of effective treatment options. Through engaging youth this study will develop transferable research methods for studying cannabis products in children.

\section{References}

1 Seshia SS, et al. Headache in Children and Adolescents: Springer; 2016. p. 85-93.

2 Seshia SS. Curr Pain Headache. Rep. 2012;16(1):60-72.

3 Lipton RB, et al. Headache. 1994;34(6):319-28.

4 Bille BS. Acta Paediatr Suppl. 1962;136:1-151. 
10

\section{Knowledge, Attitude, and Perception of Pakistani Anesthesiologists About Medical Usage of Cannabis for Pain}

R.I. Khan, G. Afshan

Aga Khan University, Anaesthesiology, Karachi, Pakistan

Introduction: Medical literature reflects acceptance of Cannabis as a medicinal drug. Pakistan ranks second amongst the countries using cannabis for recreational usage. However, medical use of Cannabis is not common. Cannabis is generally considered a recreational drug and acceptance of its medicinal use requires a cultural acceptance. Anecdotally, a few pain physicians are prescribing the very expensive imported formulations for cancer pain or other forms of chronic pain. We have devised a KAP (knowledge, attitude, practice) survey about the medicinal use of Cannabis, to find out potential barriers to its use, and to establish a baseline health-related behavior. Methodology: After ethics review committee approval, anesthesiologists working in centers that provide pain management services will be contacted through email and requested to fill an online KAP survey. This survey will contain questions relevant to the knowledge about; available preparations of Cannabis, legality of use in the country, indications and contraindications, side effects, Attitude; willingness to use if available, barriers to use, and Practice; their experience of using medical Cannabis. Survey will be administered through Google forms. Selected participants will be sent a reminder after 10 days of the first email, and a second reminder after 20 days. Data will be analyzed by Statistical packages for social science version 17 (SPSS Inc., Chicago, IL). Mean and standard deviation will be computed for age and experience of anesthesiologist. Frequency and percentage will be computed for gender, knowledge, attitude and perception of Pakistani anesthesiologists about medical usage of cannabis. Chisquare test or Fisher exact test will be used at $\mathrm{p} \leq 0.05$ level of significant.

11

\section{Effects of CBD on Diabetes and Lipid and Kidney Function}

R. Love ${ }^{1}$, C. Thirlwell

${ }^{1}$ Harvest Medicine, Family Medicine, Calgary, Canada; ${ }^{2}$ Sleep

Wake Awareness Program, Sleep Medicine \& Psychiatry, North

York, Canada

Cannabis medicine often amazes the user and the prescriber with unanticipated benefits. We have seen patients who have come in for help with arthritis and noticed improvement in bladder function, patients coming for help with depression and their blood pressure normalizes, and so on. This is a case study of a 77 year old man who presented for treatment of his osteoarthritis and was found to have significant improvement in his diabetes, lipids and kidney function with no other change in confounding factors such as diet and exercise. The patient protocol was to start on CBD oil at $5 \mathrm{mg}$ TID and titrate to $20 \mathrm{mg}$ TID in 2 weeks. He took a balanced oil 1:1 THC:CBD at bedtime for sleep as needed. He started at $0.1 \mathrm{mls}$ of this and titrated up to $0.3 \mathrm{mls}$ at bedtime as needed. The treatment was under the supervision of his family physician and his cannabis physician. We review his results at 3-month intervals from initiation of treatment. At the one year mark of being on CBD oil, the patient decreased from $1500 \mathrm{mg}$ Metformin daily to zero. His atorvastatin decreased from $20 \mathrm{mg}$ to $10 \mathrm{mg}$. He lost 15 pounds in total. His joint pain was markedly reduced and he reported increased activities of daily living and improved sleep. The patient was so impressed by his results that he felt he did not need CBD any longer and discontinued on his own. Within 4 months, we noticed that his $\mathrm{HbA1C}$ had climbed back up to $6.6 \%$, although his LDL stayed stable at $1.73 \mathrm{mmol} / \mathrm{L}$, urine ACR was $3.11 \mathrm{mg} / \mathrm{mmol}$. Due to cost issues, he restarted on Metformin but there was no decrease in his sugars, lipids or kidney function. However, when he restarted CBD at $20 \mathrm{mg}$ TID once again, within 3 months, his HbA1C, LDL and urine ACR all decreased again showing a direct correlation between such unanticipated benefits and CBD use.

\section{2}

\section{Cannabis Medicine: A Paradigm Shift from Evidence-Based Medicine to Patient-Based Practice}

R. Love ${ }^{1}$, C. Thirlwell ${ }^{2}$

${ }^{1}$ Harvest Medicine, Family Medicine, Calgary, Canada; ${ }^{2}$ Sleep

Wake Awareness Program, Sleep Medicine \& Psychiatry, North

York, Canada

One of the biggest concerns for opponents of cannabis medicine is the supposed "lack of evidence". We are often told that the patient reported outcomes are "anecdotal" and too small in numbers to support stronger global implementation for use in medical conditions and for policy formation. We have seen over 30,000 patients at our clinics for a variety of conditions ranging from chronic pain and anxiety to fibromyalgia and irritable bowel syndrome. The patients are assessed every three months and their responses to cannabis medicine are monitored. This presentation looks at the self-reported patient outcomes for a variety of medical conditions for thousands of patients spread over two years. It is compelling data that is significantly different and more positive than patient responses to many pharmaceutical medicines. These clinic-based results highlight the importance of initiating patientbased practice in cannabis medicine and using these clinical findings to inform further research in the field of cannabis medicine. The old norms for evidence-based clinical practice and conducting research will not adequately address the healing and harm reduction potential of cannabis medicine and new paradigms and strategies must be developed. 


\section{3}

\section{The Impact of Cannabis Tetrahydrocannabinol in 2-Arachidonoylglycerol (2-AG) Metabolic Enzymes in Placenta}

J.Maia ${ }^{1}$, B. Fonseca ${ }^{1}$, S. Cunha ${ }^{2}$, J. Braga ${ }^{3}$, D. Gonçalves ${ }^{3}$, N. Teixeira' ${ }^{1}$, G. Correia-Da-Silva ${ }^{1}$

${ }^{1}$ UCIBIO, REQUIMTE, Biological Sciences, Porto, Portugal; ${ }^{2}$ LAQV, REQUIMTE, Departamento De Ciências Químicas, Porto, Portugal; ${ }^{3}$ Centro Materno-Infantil do Norte-Centro Hospitalar do Porto, Departamento Da Mulher E Da Medicina Reprodutiva, Porto, Portugal

The endocannabinoid system (ECS) is composed by the cannabinoid receptors $\mathrm{CB} 1$ and $\mathrm{CB} 2$, their main endogenous ligands $\mathrm{N}$-arachidonoylethanolamine (AEA) and 2-arachidonoylglycerol (2-AG) as their main metabolic enzymes of synthesis and degradation: $N$-acylphosphatidylethanolamide-specific phospholipase D (NAPE-PLD) and fatty acid amide hydrolase (FAAH) for AEA and diacylglycerol lipase (DAGL) and monoacylglycerol lipase (MAGL) for 2-AG. Among the physiological functions modulated by the ECS, the reproductive one has been a hot topic, given the importance of a tight regulation of the endocannabinoid levels on decidualization and placentation. Moreover, the increase in cannabis consumption by woman during gestation has led to pour pregnancy outcomes such as low birth weight, prematurity and intrauterine growth restriction. These effects may be due to the main psychoactive compound of cannabis, Tetrahydrocannabinol (THC), since, even though it is hard to prove its effect in cannabis consumers, given the plethora of compounds present in the plant, we recently presented evidence that THC impairs the AEA levels and its metabolic enzymes in placental explants. To provide a better understand of the biochemical mechanisms behind these pour outcomes, we studied the impact of THC in 2-AG metabolism by quantification of its levels and expression of its main metabolic enzymes DAGL and MAGL in placental explants. Our results showed that both DAGL and MAGL are present in the placental explants in the syncytiotrophoblast layer. THC caused an increase in both DAGL and MAGL after short periods, while a decrease in MAGL expression was observed for longer periods of incubation. However, 2-AG levels remained unchanged in both periods of culture, suggesting that the 2-AG levels may be controlled by other mechanisms that are currently under evaluation. Acknowledgments: Work financed by FEDER through COMPETE and FCT through PTDC/DTP-FTO/5651/2014-POCI01-0145-FEDER-016562; FCT/MEC and FEDER, under PT2020 (UID/01/0145/FEDER/007728) and CCDR-N/NORTE2020/Portugal 2020 (norte-01-0145-FEDER-000024). Sara Cunha also acknowledges FCT for IF/01616/2015 contract. João Maia also thanks FCT for the PhD grant BD/136105/2018.

\section{4}

Effects of CBD-Enriched Cannabis Sativa Extract on Autism Spectrum Disorder Symptoms: An Observational Study of 18 Participants Undergoing Compassionate Use

P. Flaury' ${ }^{1}$, F. Caixeta' ${ }^{2}$, J. Brasil' ${ }^{2}$ L. Ramires Da Silva ${ }^{3}$, R. Malcher-Lopes $^{2}$

${ }^{1}$ ePrimeCare Healthcare SA, Brazil, Do Not Apply, Beolo Horizonte, Brazil; ${ }^{2}$ UnB, Department of Physiologicl Sciences, Brasilia, Brazil; ${ }^{3}$ Clinical Hospital, Federal University of Minas Gerais, Brazil, Mastology, Belo Horizonte, Brazil

Autism Spectrum Disorders comprise conditions that may affect cognitive development, motor skills, social interaction, communication and behavior. This set of functional deficits often results in lack of independence for the diagnosed individuals, and severe distress for patients, families and caregivers. There is a mounting body of evidence indicating the effectiveness of pure cannabidiol (CBD) and $\mathrm{CBD}$-enriched Cannabis sativa extract (CE) for the treatment of autistic symptoms in refractory epilepsy patients. There is also increasing data support for the hypothesis that non-epileptic autism shares underlying etiological mechanisms with epilepsy. Here we report an observational study with a cohort of 18 autistic patients undergoing treatment with compassionate use of standardized CBD-enriched CE (with a CBD to THC ratio of 75/1). Among the 15 patients who adhered to the treatment (10 non-epileptic and 5 epileptic) only one patient showed lack of improvement in autistic symptoms. Due to adverse effects, three patients discontinued CE use before one month. After 6-9 months of treatment, most patients, including epileptic and non-epileptic, showed some level of improvement in more than one of the eight symptom categories evaluated: Attention Deficit / Hyperactivity Disorder; Behavioral Disorders; Motor Deficits; Autonomy Deficits; Communication and Social Interaction Deficits; Cognitive Deficits; Sleep Disorders and Seizures, with very infrequent and mild adverse effects. The strongest improvements were reported for Seizures, Attention Deficit / Hyperactivity Disorder, Sleep Disorders and Communication and Social Interaction Deficits. This was especially true for the 10 non-epileptic patients, nine of which presented improvement equal to or above $30 \%$ in at least one of the eight categories, six presented improvement of $30 \%$ or more in at least two categories and four presented improvement equal to or above $30 \%$ in at least four symptom categories. Ten out of the fifteen patients were using other medicines, and nine of these were able to keep the improvements even after reducing or withdrawing other medications. The results reported here are very promising and indicate that CBD-enriched CE may ameliorate multiple ASD symptoms even in non-epileptic patients, with substantial increase in life quality for both ASD patients and caretakers.
CannX: The International Medical Cannabis Conference Lisbon 


\section{5}

\section{Raman Spectroscopy for the Characterization of Active Compounds from Cannabis Sativa Essential Oil and Determination of their Hepatoprotective Properties}

D.S. Naz

University of Education Lahore, Division of Science and

Technology Chemistry Department, Multan, Pakistan

In this study the steam extracted pure essential oil of hemp (Cannabis sativa $\mathrm{L}$ ) as well as its different fractions, obtained by fractional distillation of oil, were analyzed using Raman spectroscopy coupled with the Principal Component Analysis (PCA), a multivariate data analysis technique. The most important features observed in the Raman spectra are used to identify the presence of different components in the pure hemp oil as well as in its different fractions. These Raman spectral features can be considered as markers to provide information about the chemical composition of the hemp essential oil. Principal Component analysis (PCA) is employed to identify the biochemical features responsible for the differentiation between different fractions of hemp essential oil. Hepatoprotective activity of Cannabis sativa (Hemp) essential oil and its different fractions was studied using a carbon tetrachloride induced liver fibrosis model in rats. The hepatotoxicity produced by chronic carbon tetrachloride administration was found to be inhibited by Cannabis sativaessential oil and its different fractions with the evidence of decreased levels of serum aspartate aminotransferase, alanine aminotransferase, alkaline phosphatase and bilirubin. The histopathological findings also suggest that Cannabis sativaessential oil prevents the development of chronic liver damage. The changes in the body weights in the rats assigned to the study groups supported these biochemical and histopathological findings. The results of this study clearly indicate that Cannabis sativaessential oil and its five different fractions have a potent hepatoprotective action against carbon tetrachloride-induced liver fibrosis in rats. The fraction- 5 was found to have maximum percentage/concentration of the active component of $\beta$-carophyllene indicated by Raman spectroscopy. Moreover, these findings are much consistent with the results of the hepatoprotective assay indicating maximum activity dueto the treatment of this fraction of hemp essential oil in the rat models.

\section{6}

\section{Marijuana - Is It a Medicine?}

T. Oberbarnscheidt ${ }^{1}$, N. Miller $^{2}$

${ }^{1}$ University of Pittsburgh, 3501 Forbes Avenue, Pittsburgh, United States of America; ${ }^{2}$ Health Advocates PLLC, Psychiatry, East Lansing, United States of America

This review is based on literature search to determine the risks and benefits of marijuana as a medical agent and the role for marijuana in mainstream medicine. "Marijuana" is the natural form of cannabis derived from the cannabis sativa plant and refers specifically to the dried leaves and flowering tops or "herbal cannabis". Other terms for marijuana are cannabis or hemp. Current clinical studies do not focus on the natural form of marijuana, but evaluate synthetical forms of THC instead. Marijuana is a schedule I substance by federal law in the US with high potential of addiction and no medical benefit which limits research studies and forbids clinical use. However, the synthetical form THC can not be compared with the natural form of marijuana and vice versa. Unlike most medications on the market, marijuana does not have only one or two active agents. Natural forms of marijuana is derived from the plant, cannabis sativa, and consists of over 400 compounds including flavonoids and terpenoids and more than 60 cannabinoids other then delta-tetrahydrocannabinol (THC). Many of these compounds are not understood and their effects and adverse effects unknown. This systematic review of literature is looking at the various studies for medical conditions currently used in context of "medical" marijuana.

\section{7}

\section{Marijuana's Effect on the Adolescent}

\section{T. Oberbarnscheidt ${ }^{1}$, N. Miller $^{2}$}

'University of Pittsburgh, 3501 Forbes Avenue, Pittsburgh, United States of America; ${ }^{2}$ Health Advocates PLLC, Psychiatry, East Lansing, United States of America

Marijuana is the most commonly used illicit substance in the United States and worldwide. The smoking of marijuana is an increasingly observed phenomenon in the adolescent population and even more common nowadays than cigarette smoking. A special focus should therefore aim to the effects of marijuana in that particular age group. Adolescents are particularly vulnerable to the effects of marijuana because their brain and neuro-circuits are still developing. The exposure of marijuana to the still pruning brain causes not only short-term cognitive impairment but also permanent, life-long reduction in their cognitive abilities. This is due to marijuana's effect on processing speed and a reduction in gray matter in several brain regions as well as a decrease in white matter. Marijuana is viewed as a "gateway" drug and more so than adults, the adolescents are at higher risk to develop a subsequent drug addiction after the exposure to marijuana. A positive correlation between the age of first exposure to marijuana and the development for an addiction to other drugs has been shown. There is also a strong association between the onset of other psychiatric disorders, for example bipolar disorder, psychosis, depression and anxiety and even suicidal ideations in context with the use of marijuana. This presentation is a systematic literature review of the current scientific literature regarding the indications, toxic effects and pathological evidence from the use of marijuana in the adolescents. 


\section{8}

\section{Health Policy of Marijuana}

T. Oberbarnscheidt ${ }^{1}$, N. Miller $^{2}$

${ }^{1}$ University of Pittsburgh, 3501 Forbes Avenue, Pittsburgh, United States of America; ${ }^{2}$ Health Advocates PLLC, Psychiatry, East Lansing, United States of America

Marijuana is a substance that has been used for recreational and addictive purposes since ancient years and is currently purported to have a therapeutic or medical value and claimed to a medicine, popularly known as "medical marijuana. In the United States, marijuana is not an approved medication by the Federal Drug Administration (FDA), though currently legally available in its natural smoked and edible plant form in some states, sold as medical marijuana. According to the FDA, marijuana is classified as a Schedule I drug with high risk of addiction and dangerousness with no legitimate medical purpose. However, contrary to US federal law, marijuana is declared legal without a medical approval process in several states ostensibly for deliberating conditions e.g. various pain conditions, depression, anxiety, nail patella, glaucoma and even HIV. However, medical marijuana is also available and used more commonly for various other purposes, particularly, addictive use, even though studies have shown greater harmful effects then beneficial. With marijuana, the rate of misperception is high in the users that risks for adverse consequences are low and benefits high. While marijuana is legally available in some states and other states are pressured to follow suit, the United States House of Representatives has proposed legislation to legalize marijuana as a beverage, similar to alcohol and the United State Senate had proposed legislation to legalize marijuana as medication, reclassifying marijuana to a Schedule II drug. Marijuana appears here to stay in the US in some form, and entrepreneurs line up to profit billions from its anticipated sales and widespread distribution. This article is a systematic review of literature analyzing the current policies, medical facts, legal status, and commercial trends as well as politics regarding marijuana and its manufacture, sales, and distribution. The article is focused on the natural form of Tetrahydrocannabinol (THC) derived from the Cannabis sativa plant, and not the less sought and the actual medical cannabinoids, both natural and synthetic forms, available for medical use. Our aim to provide education, facts, and discussions which will lead to safe and healthy public policy for marijuana in whatever form it takes in the future.

\section{9}

\section{Marijuana Violence and the Law}

T. Oberbarnscheidt ${ }^{1}, \underline{\text { N. Miller }}^{2}$

${ }^{1}$ University of Pittsburgh, 3501 Forbes Avenue, Pittsburgh, United States of America; ${ }^{2}$ Health Advocates PLLC, Psychiatry, East Lansing, United States of America

Marijuana is currently a growing risk to the public in the United States. Following expanding public opinion that marijuana provides little risk to health, state and federal legislatures have begun changing laws that will significantly increase accessibility of marijuana. Greater marijuana accessibility, resulting in more use, will lead to increased health risks in all demographic categories across the country. Violence is a well-publicized, prominent risk from the more potent, current marijuana available. We present cases that are highly popularized storylines in which marijuana led to unnecessary violence, health risks, and, in many cases, both. Through the analysis of these cases, we will identify the adverse effects of marijuana use and the role it played in the tragic outcomes in these and other instances. In the analysis of these cases, we found marijuana as the single most common, correlative variable in otherwise diverse populations and circumstances surrounding the association of violence and marijuana.

\section{0 \\ Cannabidiol as Premedication in Terms of Postoperative Pain Scores After Major Orthopedic Surgical Procedures}

\section{E. Papaioannou, A. Analytis, E. Mavrommatis, G. Koziakas,}

A. Lampadariou

"Laikon" General Hospital of Athens, Anesthesiology and Pain Management, Athens, Greece

Aim of this study was the evaluation of cannabidiol (CBD) versus commonly prescribed alprazolam as ppremedication, in terms of postoperative pain. Material and Methods In a perspective randomized study 56 scheduled to total knee arthroplasty, under subarachnoid anaesthesia, were divided in two groups, Group A ( $\mathrm{n}=$ 28) patients received CBD $50 \mathrm{mg}$ capsule and group $\mathrm{B}(\mathrm{n}=28)$ alprazolam $0.5-1 \mathrm{mg}$ tablet per os, as premedication on the morning of operation. Both groups'patients received tramadol 50-100 mg x 4, and parecoxib $40 \mathrm{mg} \times 2 / 24 \mathrm{hr}$ iv. We recorded patients' demographics, operation duration, intra- and postoperative surgical complications, and pain scores postoperatively 1, 6, 12, 24, 36, 48 and 72 hours after end of subarachnoid anaesthesia. Other perioperative complications, such as nausea, vomiting, haemodynamic variations, as well as need for extra analgesics postoperatively were also recorded. Results Demographics and operation duration did not differ significantly between two groups. No surgical complications were recorded. On the first, 6th, 12th and 24th postoperatively, pain scores were significantly lower in group A, while there was no difference 36, 48 and 72 hours later. Need for extra analgesia was significantly higher in group $b$ at all times of study. 6 group $A$ and 11 group B patients demonstrated nausea and all of them were haemodynamically stable. Conclusion CBD as premedication seems to provide lower pain scores intra and 24 hours postoperatively, compared to commonly prescribed alprazolam. Further study is needed to support this conclusion.
CannX: The International Medical Cannabis Conference Lisbon 


\section{1 \\ Effects of Cannabidiol During Pregnancy in the Cognitive Development of Newborn Babies}

D. Peña, M. Junco

Universidad Anáhuac, Department of Psychology, Mexico City, Mexico

One of the main symptoms that is key and underlies a large amount of different neuropsychiatric disorders is mild cognitive impairment. Cannabidiol (CBD) has been used recently to treat a wide variety of health issues (childhood epilepsy, anxiety, insomnia, chronic pain). We examined and compared if CBD had any effects on cognitive processes (spatial, work and long term memory and motor coordination) with an experimental model of Sprague-Dawley rats that were sorted into two groups; control (no CBD) and experimental (CBD). Furthermore, we also examined if the use of CBD would have any effects on the neurocognitive development of newborn rats and their behavior. Both groups were composed of female rats that were crossed over with male rats in order to get them pregnant, consequently allowing us to use their offsprings as objects of study with an elevated plus maze test. A dosage of $0.3 \mathrm{ml}$ of CBD was administered to the experimental group of pregnant rats in $500 \mathrm{ml}$ of water. On the other hand, newborn rats were administered $50 \mu \mathrm{L}$ of pure CBD two weeks after being born. Our findings evidence that the CBD administered to the pregnant rats has an effect in their newborn babies; showing higher anxiety levels compared to the control group. Further research is required to determine the cause and long term effects of CBD treatment during pregnancy.

\section{2 \\ The Challenges of Transdermal Delivery of Cannabinoids}

J. Riggle

Mary's Medicinals, Science - Research \& Development, Denver, United States of America

As the therapuetic potential of cannabinoids for human health become more established and cannabinoid therapy gains broader acceptance as a legitimate form of treatment many new questions emerge. Which cannabinoid is best suited for what specific indication? What is the most efficient route of administration? What is the most effective dose? All of these questions address important details that need to be resolved for appropriate application of cannabinoid therapy to become a reality. Different routes of administration all come with significant limitations and disadvantages. For example, first-pass hepatic metabolism following oral administration presents a challenge when trying to provide accurate dosing. Inhalation of any type of burned organic material exposes patients to carcinogenic pyrolysis byproducts. In addition, the variable and relatively low bioavailability of cannabinoids via these different routes of administration all further contribute to the complications associated with the application of cannabinoid therapy. Transdermal delivery of certain active pharmaceutical ingredients (APIs) - fentanyl and progesterone for example - has been shown to be an effective form of drug delivery. The potential of transdermal delivery of cannabinoids offers an alternative method for patients seeking relief that avoids many of the complications and risks mentioned above. However, due to the protective nature of the stratum corneum, transdermal drug delivery is not a trivial matter and presents numerous challenges of its own. This presentation will focus on the nature of the stratum corneum, the associated challenges and the different approaches developed to overcome these obstacles. The latest research on transdermal cannabinoid delivery, including efficacy, formulations, specific products - transdermal patches and creams for example - and overall results will be discussed. The pharmacokinetics and pharmacodynamics of transdermal cannabinoid delivery will also be covered as well as ideal indications for the application of transdermal cannabinoid therapy. Finally, a number of human case studies utilizing transdermal delivery of cannabinoids will be presented and described.

\section{3 \\ The Endocannabinoid System of the Skin and Its Potential in Skin Indications}

\section{$\underline{\text { J. Riggle }}$}

Mary's Medicinals, Science - Research \& Development, Denver, United States of America

The Endocannabinoid System (ECS), discovered at the end of the twentieth century, is a G-protein coupled receptor system involved in a number of physiological processes including but not limited to: pain sensation, metabolism, immunomodulation and inflammation. The ECS consists of cannabinoid receptor subtype $1\left(\mathrm{CB}_{1}\right)$, primarily found in the central nervous system, and cannabinoid receptor subtype $2\left(\mathrm{CB}_{2}\right)$, found predominantly in the lymphatic system. In addition to $\mathrm{CB}_{1}$ and $\mathrm{CB}_{2}$ receptors, there are also endocannabinoids which are produced by the body, with 2-arachidonoylglycerol (2-AG) and $\mathrm{N}$-arachidonoylethanolamine (AEA) being the most well characterized. The enzymes responsible for the synthesis and degradation of endocannabinoids also play a significant role in the ECS. Phytocannabinoids, compounds unique to the cannabis plant, have been found to modulate and influence ECS activity, with the two most well studied molecules being $\Delta$-9-Tetrahydrocannabinol (THC) and Cannabidiol (CBD). This receptor system, including the endocannabinoids and their synthesis/degradation enzymes, can be found in virtually all human skin cell types, indicating a significant role of the ECS in skin pathophysiology. Basic research has shown tremendous potential for treating various skin conditions with cannabinoids. In this presentation, animal model and preclinical studies demonstrating the potential role of cannabinoids in treating different skin conditions from pain and itch to acne will be described. The challenges and obstacles to their use in topical and cosmetic products, including regulatory hurdles, chemical compatibility and formulation issues will be discussed. Specific cannabinoids for certain indications and appropriate dosing will also be covered. 


\section{4}

\section{Treating Diabetes Mellitus with Cannabis - From Lab to the Clinic}

D. Robinson ${ }^{1}$, H. Robinson ${ }^{2}$, M. Yassin ${ }^{1}$

${ }^{1}$ Hasharon Hospital, Orthopedics, Petah Tikwa, Israel;

${ }^{2}$ University of Nicosia, Md Progarm, Nicosia, Cyprus

Medical cannabis usage has spread over the last few years in jurisdictions were it has been permitted, despite the plant being defined as a schedule one drug. Due to a fairly large body of evidence demonstrating possible amelioration of neuropathy symptoms following cannabis usage, this indication is currently approved in our nation. Patients must undergo a minimum of 12 months treatment by other medications in a pain clinic. The current cohort is a sequential cohort of 89 diabetic patients who failed a minimal 12 months course of drug therapy. The patients were treated by at least three drugs prior to instigation of medical cannabis therapy. The cannabis therapy was a smoked preparation with a minimal THC content of 10 percent and minimal CBD content of 2 percent. The dose was increased as necessary after a minimum of 3 months of therapy. Prior to therapy the patients had performed the following blood tests: hsCRP, total cholesterol, nonHDL cholesterol, HDL and complete blood count. These tests were repeated after 12 months of therapy. The primary outcome measure was the NRS and it declined from $8.7 \pm 1.1$ to $4.1 \pm 3.1$ post 24 months cannabis therapy. The BPI score decreased from $7.8 \pm$ 2 to $2.9 \pm 1$. The CRP averaged $1.4 \pm 0.8$ and decreased to $0.6 \pm 0.3$. Hemoglobin levels did not change. WBC levels declined (within the normal range from $9700 \pm 2500$ to $6550 \pm 2100$ ). Average blood glucose at baseline was $165 \pm 26$ and declined to $129 \pm 25$ at 24 months. HbA1C declined an average of 2.4 grams per dl. It is possible to conclude that cannabis therapy has an anti-inflammatory effect in diabetic patients with neuropathy as well as an analgesic one it is paralleled by improvement in glycemic balance. To investigate the role that CBD plays in this effect, 20 Wistar female rats were treated for one month with fattening diet. Hyperglycemia was induced as demonstrated by fasting glucose levels over $150 \mathrm{mg} / \mathrm{dl}$. Then intraperitoneal CBD injections were performed $(1 \mathrm{mg} / \mathrm{kg}$ body weight). CRP levels declined but glycemic control was not achieved using CBD injections. We conclude that the full entourage effect is necessary to replicate in rats the human results.
25

An Observational Study on the Treatment of Eczematous Conditions with a Topical Cannabinoid Gel Designed to Improve the Appearance of Affected Skin

H. Yardley' ${ }^{1}$, C. Rundle ${ }^{2}$, S. Dercon ${ }^{3}$, P. Lio ${ }^{4}$, J. Fernandez ${ }^{1}$, M. Fujita ${ }^{2}$, R. Dellavalle $^{2}$

${ }^{1}$ Altus Labs, Development, Denver, United States of America; ${ }^{2}$ University of Colorado, Dermatology, Aurora, United States of America; ${ }^{3}$ University of Southern California Keck School of Medicine, School of Medicine, Los Angeles, United States of America; ${ }^{4}$ Northwestern University Feinberg School of Medicine, Dermatology, Chicago, United States of America

Introduction: Atopic Dermatitis (AD) is an exceptionally common disease, affecting up to $20 \%$ of children and up to $3 \%$ of adults worldwide. AD can significantly compromise patients' quality of life by causing sleep disturbance, itch, secondary infection, frustration and anxiety, as well as distressing cosmetic signs of disease. Itch, or pruritus, is a hallmark feature of AD. The impact of ADrelated pruritus can range from mildly irritating or distracting to completely disabling. Traditionally, management of itch in AD patients has focused on restoring the altered skin barrier with topical emollients, reducing inflammation, restoring microbiome balance, or focusing on the central itch signaling pathways. The endocannabinoid system mediates many of these pathways, and this has turned researchers' attention towards this system and exogenous cannabinoids for novel targets in treating AD. Methods: This is an observational study of individuals using a CBD-infused topical to treat their eczematous skin conditions. The study population consisted of 14 subjects ( $\mathrm{n}=11$ male; mean age $=51.5 \pm 16.7$ years $)$ with an eczematous skin condition, as diagnosed by a dermatologist. Subject's eczema severity and itch intensity was assessed prior to and after 2 weeks of ad libitum CBD topical usage. EASI scores and the 5D Itch Scale were used. Results: The CBD topical significantly reduced EASI scores by $41 \%$ overall $(\mathrm{p}<0.05)$, reduced itch in $57 \%$ of subjects, with an average reduction of $33 \%$ for the $5 D$ Itch Scale $(p=0.05)$ in subjects that experienced a reduction in itch. VAS itch scores showed an average trend in reduction of $29 \%$ $(p=0.06)$. Conclusion: Current approaches to treating AD such as emollients, corticosteroids and topical calcineurin inhibitors, leave much to be wanted. Comparatively, cannabinoids such as CBD are non-toxic while providing a therapeutic effect for these individuals. Cannabis has an exceedingly high safety profile, presenting a novel opportunity for a low risk option for reducing the appearance of AD afflicted skin. Given the increasing awareness of these compounds and their potential benefits, more research is needed to understand the effects of these compounds in skin conditions. 


\section{6}

An Examination of Digital Approaches for Exploring the Medical Applications of Cannabinoids: Evaluating the Impact of Topical CBD on Atopic Dermatitis

H. Yardley ${ }^{1}$, C. Rundle ${ }^{2}$, S. Dercon ${ }^{3}$, P. Lio ${ }^{4}$, J. Fernandez' 1 , M. Fujita ${ }^{2}$, R. Dellavalle ${ }^{2}$

${ }^{1}$ Altus Labs, Development, Denver, United States of America; ${ }^{2}$ University of Colorado, Dermatology, Aurora, United States of America; ${ }^{3}$ University of Southern California Keck School of Medicine, School of Medicine, Los Angeles, United States of America; ${ }^{4}$ Northwestern University Feinberg School of Medicine, Dermatology, Chicago, United States of America

Introduction: Designing and running federally funded cannabis studies in the United States is a complicated process with numerous hurdles ranging from FDA approval to acquisition of federally approved cannabis from the National Institute for Drug Abuse. Using a digital platform to collect data on cannabis usage and its impact upon chronic medical conditions will give insight into the potential therapeutic uses for cannabis. Traditionally, management of itch in $\mathrm{AD}$ patients has focused on restoring the altered skin barrier with topical emollients, reducing inflammation, restoring microbiome balance, or focusing on the central itch signaling pathways. The endocannabinoid system mediates many of these pathways, and this has turned researchers' attention towards this system and exogenous cannabinoids for novel targets. Here, we evaluate the impact of a CBD topical cream on the psychological and physical manifestations of AD. Methods: This is an observational study of individuals using a CBD-infused topical to treat $\mathrm{AD}$. The study population consisted of 16 subjects recruited from online support groups who reported a previous diagnosis of $\mathrm{AD}$. The initial severity of the subject's eczema was evaluated initially using the Patient Oriented Eczema Measurement (POEM) survey and the Quality of Life in Hand Eczema Questionnaire (QOLHEQ). Patients were mailed a sample of the topical cream to apply for 2 weeks ad libitum. The severity of the eczema after this treatment period was evaluated again using the POEM and QOLHEQ scores. Results: Use of the CBD topical significantly reduced the average POEM score by $60.0 \%(\mathrm{p}<0.001)$ and the average QOLHEQ score by $48.4 \%$ ( $\mathrm{p}<0.001)$. The use of a digital survey allowed us to collect critical data regarding change in prescription medication, perceived level of itchiness, and general belief that the condition had improved. Conclusion: With measurable anti-itch, anti-pain, anti-microbial and anti-inflammatory properties, the effect of cannabinoids in patients with $\mathrm{AD}$ has already begun to be demonstrated, but more research is needed to fully understand the risks and benefits of individual cannabinoids. This study demonstrates that using a digital platform to gather data offers an effective means for further understanding the potential uses of cannabinoids.

\section{7}

\section{Anti-Inflammatory Effect of Cannabidiol and Palmitoylethanolamide on Skin in a 12-0-Tetradecanoylphorbol-13-Acetate Dermatitis Model in Mice}

\author{
H. Yardley ${ }^{1}$, C. Rundle ${ }^{2}$, S. Dercon ${ }^{3}$, R. Dellavalle ${ }^{2}$, P. Lio ${ }^{4}$, \\ J. Fernandez ${ }^{1}$, M. Fujita ${ }^{2}$
}

${ }^{1}$ Altus Labs, Development, Denver, United States of America; ${ }^{2}$ University of Colorado, Dermatology, Aurora, United States of America; ${ }^{3}$ University of Southern California Keck School of Medicine, School of Medicine, Los Angeles, United States of America; ${ }^{4}$ Northwestern University Feinberg School of Medicine, Dermatology, Chicago, United States of America

Introduction: Atopic dermatitis (AD) is an inflammatory, relapsing skin condition with a complex, multifactorial etiology that is characterized by chronically dry skin, pruritus, and eczematous lesions. Its pathogenesis is associated with alterations to both the skin barrier and the immune system. The endocannabinoid system mediates many of the pathways underlying $\mathrm{AD}$, and this has turned researchers' attention towards this system and exogenous cannabinoids for novel targets in treating AD. Recent studies have shed light on the mechanisms underlying the therapeutic effect of individual cannabinoids, but precise combinations of cannabinoids and cannabimimetic compounds have not yet been studied. Here, we use a TPA model to assess the anti-inflammatory effects of a commercially available CBD topical preparation. Results: Change in left-right ear caliper measurement differentials peaked $8 \mathrm{~h}$ postchallenge in all TPA groups with Vehicle (TPA) control animals showing a mean increase of $0.28 \mathrm{~mm}$ from baseline $(0 \mathrm{~h})$. Increases in ear thickness from baseline were inhibited $24-48 \mathrm{~h}$ post-challenge in mice treated with CQuell as compared to TPA control mice. Treatment with Mometasone Furoate Ointment significantly inhibited ear swelling from $8-24 \mathrm{~h}$ and $8-48 \mathrm{~h}$ post-challenge ( $\mathrm{p}<0.001 ; \mathrm{p}<0.001)$ as well as compared to TPA control animals at $24 \mathrm{~h}$ and $48 \mathrm{~h}(\mathrm{p}<0.001 ; \mathrm{p}<0.001)$. Treatment with CQuell also significantly inhibited ear swelling at $8-24 \mathrm{~h}$ and $8-48 \mathrm{~h}$ post-challenge ( $p<0.001 ; p<0.001)$ as well as compared to TPA control animals at $24 \mathrm{~h}$ and $48 \mathrm{~h}(\mathrm{p}=0.013 ; \mathrm{p}=0.002)$. Treatment with CQuell reduced inflammation of the ear by $51.23 \%$ at $24 \mathrm{~h}$ and $65.61 \%$ at $48 \mathrm{~h}$ post application. Treatment with Mometasone reduced inflammation of the ear by $89.82 \%$ at $24 \mathrm{~h}$, and $95.25 \%$ at $48 \mathrm{~h}$. Natural reduction in inflammation in the TPA animals was $26.32 \%$ at $24 \mathrm{~h}$ and $44.21 \%$ at $48 \mathrm{~h}$. Conclusion: Cannabinoids represent an exciting prospect for the future of $\mathrm{AD}$ therapy. With measurable anti-itch, anti-pain, anti-microbial and anti-inflammatory properties, the effect of cannabinoids in patients with $\mathrm{AD}$ has already begun to be demonstrated, but more research is needed to fully understand the risks and benefits of the individual cannabinoids present in cannabinoid products on the market. 


\section{8}

\section{Unifying Disparate Data Sources to Maximize Real World Evidence}

\section{P. Sekar}

CB2 Insights, Head Office, Mississauga, Canada

With over 40 countries and millions of patients benefiting from medical cannabis across the world every day, how can you leverage the hundreds of millions of data points to gain meaningful, clinically-validated insight? Countless disparate technology systems require a streamlined approach to collecting and analyzing this data in order for the industry to truly understand the impact that medical cannabis has on the healthcare system and the patients it serves. In this session, we will delve into what each stakeholder needs to keep in mind as the industry continues its growth and begins to merge with traditional healthcare practices.

\section{9 \\ Solving the Dilemma of Polypharmacy with Patient-Based Medical Cannabis Regimens: Harm Reduction in the Canadian Veteran Population \\ C. Thirlwell' ${ }^{1}$ R. Love ${ }^{2}$, R. Rivas ${ }^{3}$, S. Greenspan ${ }^{1}$, H. Shulman ${ }^{1}$ \\ 'Sleep Wake Awareness Program, Sleep Medicine \& Psychiatry, North York, Canada; ${ }^{2}$ Harvest Medicine, Family Medicine, Calgary, Canada; ${ }^{3}$ Vitality Health Centre Inc, Integrative \\ Medicine, Toronto, Canada}

Polypharmacy is a major issue in the Canadian Veteran population. In addition to PTSD, veterans suffer from multiple mental and physical health issues, commonly including: major depressive disorder, insomnia, chronic pain, tinnitus, irritable bowel syndrome, erectile dysfunction, post concussion syndrome, brain injury, chronic fatigue and others. These complex clinical presentations often result in the prescription of polypharmacy, which is not adequately monitored. Iatrogenic effects of multiple prescription medications, drug-drug interactions, and adverse side effects of prescription medications are common. Furthermore, there are no evidence-based clinical trials, which demonstrate safety and efficacy of combining multiple drugs across multiple physical and mental health diagnoses. Many veterans are prescribed combinations of anti-depressants, psychotropics, hypnotics, NSAIDS, opioids, and other classes of medications. Often, veterans report having been medicated with polypharmacy both during and after active military service with polypharmacy ranging from 3 to over 10 different prescription medications. Clinically, these polypharmacy regimens cause harm by further undermining physical and mental function and are associated with iatrogenic issues, such as: cognitive dysfunction, emotional numbing, anger outbursts, suicidal ideation, gastroesophageal reflux disease, erectile dysfunction, irritable bowel syndrome, obesity, central and obstructive sleep apnea, metabolic syndrome, type II diabetes, liver function abnormalities, kidney function abnormalities, and others. Medical Cannabis (MC) is a medication, which is proving to be increasingly effective across physical and mental health diagnostic categories without the adverse side effects of other prescription medications.
$\mathrm{MC}$ is a key player in the re-establishment of homeostatic balance in medically and mentally ill patients. Using a patient-based approach, we have developed personalized MC regimens in the veteran population, which allow for the weaning down of and discontinuation of prescription medications that have proved to be ineffective and/or caused adverse side effects. Instituting personalized MC regimens is proving to be a clinically effective harm reduction strategy in: 1) the reduction of polypharmacy, 2) the optimization of physical and mental health symptom management, and 3) improved physical and psychosocial function.

\section{0}

Spontaneous, Anecdotal, Retrospective, Open-Label Study on the Efficacy, Safety and Tolerability of Cannabis Galenical Preparation (Bedrocan)

\section{Vadalà}

University of modena, Surgery, modena, Italy

Objectives: Our main aim was to investigate the short-term therapeutic effects, safety/tolerability and potential side effects of the cannabis galenical preparation (Bedrocan) in patients with a range of chronic conditions unresponsive to other treatments. Methods: In this retrospective, 'compassionate use', observational, open-label study, 20 patients (age 18-80 years) who had appealed to our 'Second Opinion Medical Consulting Network' (Modena, Italy), were instructed to take sublingually the galenical oil twice a day for 3 months of treatment. The usual starting dose was low $(0.5 \mathrm{ml} /$ day $)$ and gradually titrated upward to the highest recommended dose $(1 \mathrm{ml} /$ day). Tolerability and adverse effects were assessed at baseline and monthly thereafter during the treatment period through direct contact (email or telephone) or visit if required. Patients' quality of life was evaluated at baseline and 3 months using the medical outcome short-form health survey questionnaire (SF-36). Key Findings: From baseline to 6 months posttreatment, SF-36 scores showed: reductions in total pain $(\mathrm{P}<0.03)$; improvements in the physical component $(\mathrm{P}<0.02)$; vitality $(\mathrm{P}<$ $0.03)$; social role functioning $(\mathrm{P}<0.02)$; and general health state $(\mathrm{P}<0.02)$. No changes in role limitations $(\mathrm{P}=0.02)$ due to emotional state (e.g. panic, depression, mood alteration) were reported. Monthly reports of psychoactive adverse effects showed significant insomnia reduction $(\mathrm{P}<0.03)$ and improvement in mood $(\mathrm{P}<0.03)$ and concentration $(\mathrm{P}<0.01)$. Conclusions: These data suggest that a cannabis galenical preparation may be therapeutically effective and safe for the symptomatic treatment of some chronic diseases. Further studies on the efficacy of cannabis as well as cannabinoid system involvement in the pathophysiology are warranted. 


\section{1}

\section{A Therapeutic Effect of CBD-Enriched Ointment in Inflammatory Skin Diseases and Cutaneous Scars \\ M. Vadalà}

University of Modena, Surgery, Modena, Italy

Objective: To investigate the therapeutic effect of CBD-ointment administered on severe skin chronic diseases and/or on their outcome scars. Methods: A spontaneous, anecdotal, retrospective study of 20 patients with two most frequent skin disorders: psoriasis (n: 5 patients), atopic dermatitis (n: 5) and resulting outcome scars (n: 10). The subjects were instructed to administer topical CBD-enriched ointment to lesioned skin areas twice daily for three months treatment. Results: Based on skin evaluations (hydration, TEWL, elasticity), clinical questionnaires (SCORAD, ADI, PASI), and supported by photographic data and investigators' clinical assessment, the results showed that topical treatment with CBD-enriched ointment significantly improved the skin parameters, the symptoms and also the PASI index score. No irritant or allergic reactions were documented during the period treatment. Conclusions: The topical administration of CBD ointment, without any $\mathrm{THC}$, is a safe and effective non-invasive alternative for improve the quality of life in patients with some skin disorders, especially on inflammatory background.

\section{2 \\ Medical Cannabis as a Therapeutic Resource: Preliminary Study}

P.M. Velazquez Ramos, J. Galzerano

Prívate Clinical, Medical Cannabis, Montevideo, Uruguay

Introduction: Cannabis Sativa L. products are known to be therapeutically effective since ancient times, despite its pharmacological typification was made in the mid-20th century. Its use was recently regulated in Uruguay. Objective: To analyse a preliminary clinical-therapeutic experience with MC with high content of cannabidiol (CBD). Method: Epidemiological, observational and retrospective study of a 355 patient cohort who spontaneously consulted to learn about CM at a private clinic, between August 2016 and December 2017. Demographic data, medical records, expectation and previous experience with cannabis were collected in the first interview. In most cases, cannabis with high content of CBD was prescribed (5.25\% CBD and $0.2 \% \mathrm{THC}$ ). In subsequent consultations, access to access to $\mathrm{MC}$ was investigated and both response to it and adverse effects were studied by means of analogue scales. The study used descriptive statistics. Results: In the cohort studied, women with an average age of 67 years old and university studies prevailed. The following conditions motivated consultations: neurological (38\%), rheumatic or bone degenerative diseases (37\%), neoplasms (13\%), psychiatric diseases (4\%) and miscelánea (8\%). In most cases $(60.6 \%)$ patients stated symptoms improved and only $16.3 \%$ of the populaiton studied presented mild adverse effects. High costs and difficulties in accessing MC were the reasons for not starting or abandoning treatment. Conclusions: Our preliminary study reflects the positive therapeutic response and non significant adverse effects to $\mathrm{MC}$ with high content of CBD. $60.6 \%$ of patients treated referred improvement in their symptoms. Decisive factors for a successful treatment point at the need to make access to MC easier by improving management to obtain it and reducing costs for a greater accessibility.

\section{Business: New Technologies, New Opportunities}

\section{3 \\ Usage of Medical Cannabis in Elderly Patients with Chronic Pain Associated Diseases}

1. Gago-Rodrigues ${ }^{1,2}$, F. Águas², J. Nascimento ${ }^{2}$, S. Pais 1,3

${ }^{1}$ Universidade do Algarve, Centro De Investigação Em Biomedicina, Faro, Portugal; ${ }^{2}$ Prisma Produtos Farmacêuticos, Lda, Investigação E Desenvolvimento, Vila Real de Santo António, Portugal; ${ }^{3}$ Universidade do Algarve, Centro De Internacional Sobre O Evelhecimento, Faro, Portugal

The use of cannabis plant for medical proposes is a millenary practice, there were found evidences of its usage in India by 1000 $\mathrm{BC}$. The pharmacological benefits and proprieties of cannabis are explore since ancient era, however its consumption was criminalized in various countries, starting in the beginning for $19^{\text {th }}$ century until nowadays. Currently, the usage of medical cannabis is becoming legal in several EU countries, including Portugal, since 2019. There are scientific evidence available in which medical cannabis is associated with positive effects and potential treatment for a broad spectrum of diseases and symptoms, including chronic rheumatic pain and lack of appetite. These diseases and symptoms are frequently associated with ageing related diseases and normally are addressed with pharmacological drugs as opioids, in the case of chronic pain, which can increase the lack of appetite in patients. Our aim is to evaluate the benefits of using medical cannabis to treat chronical pain patients, assessing the levels of pain changes and a related increase in appetite. To fulfill our objectives, we aim to use a group of elderly patients, showing osteoarthritis and rheumatoid arthritis in cohort studies to assess the effects of medical cannabis in both, the pain related disease associated symptoms, as in the appetite matters. Our studies include a multidisciplinary collaboration, including pharmaceutical research and development, patient's clinical evaluation in terms of the disease characterization and treatment; with the main goal to contribute for the improvement in life quality of senior patients suffering from chronic pain associated diseases. 


\section{4 \\ Biomaterials Delivery - A Novel Frontier for Cannabis Treatments}

l. Roversi

Vividess Precision Medicine, Ceo, Sao Paulo, Brazil

Biomaterials are a type of polymers that present the properties of biocompatibility integrating the extracellular matrix and providing a micro ambient for cell growth, developing healthy tissue in damaged areas. A particular interesting class inside this polymers are the biodegradable biomaterials, this kind of molecules have the capacity of enzymatic and hydrolytic degradation inside the human tissue. The degradation of this materials offer the possibility to release other molecules like drugs at the same time of degradation, supporting a constant dose delivery system. The drug delivery system is very useful for chronic diseases or mid and long term treatments, being applied by minimally invasive surgical technics. Cannabinoids are this kind of molecule that can be loaded in microspheres or injected inside this scaffolds to have a controlled released, maintaining a constant level of efficacy without toxicity. This research field of pharmacokinetics have some medical translational applications like patches composed by collagen matrix with CBD directed to dermal inflammations as in second and third grades burns or autoimmune diseases, hydrogels injected in subcutaneous tissue that can release THC for melanoma treatment, intracavitary applications like a uterine delivery system and intracolonic implants developed to release CBD against tumor inflammation and THC to induce apoptosis, in Central Nervous System a platelet of biodegradable biomaterial may provide intracranial controlled liberation in tumors with capsules like Glioblastoma Multiforme, a cosmetic based on microspheres can encapsulate the cannabinoids and remain on skin. The field of delivery routes in cannabis research is so important as the basic research because supply new ways to clinical applications and more effective local treatment.

\section{Agriculture: Advanced Agriculture and Production}

\author{
35 \\ High Potential Brazilian Biomes for Cannabis ssp \\ Production \\ $\underline{\text { K. Cardoso }}^{1}$, N. Batista ${ }^{1}$, D. Gonçalves ${ }^{2}$, M. Silva $^{3}$, R. Alberici ${ }^{1}$ \\ ${ }^{1}$ ETHEL-AMMER Soluções em Biotecnologia do Brasil, P\&d, \\ Mandirituba, Brazil; ${ }^{2}$ Universidade Federal de São Paulo, Dep \\ Nutrição, Santos, Brazil; ${ }^{3}$ Universidade Estadual de Campinas, \\ Centro De Biologia Molecular E Engenharia Genética, Campinas, \\ Brazil
}

Cannabis ssp is a highly productive plant with a very high economic value, great agricultural potential and elevated adaptive capacity to the most diverse biomes around the world. Cannabis is a rustic plant and therefore it needs specific production conditions to achieve high levels of product quality for the pharmaceutical, food, textile, pulp and paper, plastics, among others. Brazil is a country with continental dimensions, with over 200 million inhabitants and six different biomes: Amazon, Cerrado, Caatinga, Atlantic Forest, Pantanal and Pampa. Each biome has different edaphoclimatic and productive characteristics that allow the adaptation and development of several varieties of plants, with high productivity and speed. Being a tropical country, Brazil has high solar incidence, little cold and abundant water. Nowadays, Brazil is one of the largest producers of commodities such as soybeans, corn, cotton, meat, among other foods. The aim of this study was to identify which are the best biomes for Cannabis production in Brazil considering medicinal and industrial purposes. For this, a computational and comparative analysis of data was performed from the databases of the Brazilian Ministry of Agriculture, Cattle and Supplying (MAPA), Ministry of the Environment (MMA), Brazilian Institute for Geography and Statistics (IBGE), National Industrial Property Institute (INPE), National Institute of metrology, Standardization and Industrial Quality (INMETRO) and Brazilian Agriculture Research Corporation (Embrapa). The results showed that the Caatinga Biome presented the highest productive potential and the best climatic conditions, producing up to three crops per year; following were the Atlantic Forest, Cerrado, Amazon, Pantanal and Pampa biomes. Brazil has the potential to become one of the largest producers of Cannabis for medicinal and industrial purposes in the world, because it has the best edaphoclimatic conditions, being possible to produce in almost all the national territory. Cannabis could be an important alternative for small and medium producers as well as large producers who could incorporate it into the high productivity mechanism. Therefore, Cannabis has great potential to become an important commodity in Brazil, which will provide many benefits to the country's production chain.
CannX: The International Medical Cannabis Conference Lisbon 


\section{Medical Cannabis and Cannabinoids}

\section{Author Index}

Numbers refer to abstract numbers
Afshan, G.G. 1, 10

Águas, F. 33

Alberici, R. 35

Alcorn, J. 8, 9

Almeida, C.F. 3

Alves, P. 2

Amaral, C. 2, 3

Analytis, A. 20

Barbosa, C. 4

Batista, N. 35

Braga, J. 13

Brasil, J. 14

Caixeta, F. 14

Cardoso, K.C. 35

Correia-Da-Silva, G. 2, 3, 13

Costa, P. 4

Cunha, S. 13

De Aguiar, F.M.V.F. 4

Dellavalle, R. 25, 26, 27

Dercon, S. 25, 26, 27

Duque, V. 4

Epstein, I. 7

Fernandez, J. 25, 26, 27
Finkelstein, Y. 8

Finley, A. 9

Flaury, P. 14

Fonseca, B. 13

Fujita, M. 25, 26, 27

Gago-Rodrigues, I. 33

Galzerano, J. 32

Goldberg, D. 6

Gonçalves, D. 13, 35

Greenspan, S. 29

Harari, R. 6

Huntsman, R. 8, 9

Ibrahim, M.E.A.E. 5

Jackson, K. 6

Junco, M. 21

Katzman, M.A. 7

Kelly, L.E. 8, 9

Khan, R.I. 1

Khan, R.I.I. 10

Khan, S. 1

Koziakas, G.E. 20

Lacaze-Masmonteil, T. 8
Lampadariou, A. 20

Lewis, E. 9

Lio, P. 25, 26, 27

Love, R. 11, 12, 29

Maia, J.O. 2, 13

Malcher-Lopes, R. 14

Mavrommatis, E. 20

Miller, N.S. 16, 17, 18, 19

Moore-Hepburn, C. 8

Nascimento, J. 33

Naz, D.S. 15

Neves, F. 4

Oberbarnscheidt, T. 16, 17, 18,19

Oberlander, T. 8,9

Pais, S. 33

Palmer, A. 8

Papaioannou, E. 20

Peña, D. 21

Pitashny, P.A. 6

Ramires Da Silva, L. 14

Riggle, J.J. 22, 23

Rivas, R.E. 29
Robinson, D. 24

Robinson, H. 24

Roversi, I.D.O. 34

Rundle, C. 25, 26, 27

Sekar, P.P. 28

Shulman, H. 29

Siddiqi, S. 1

Silva, A. 4

Silva, M. 35

Sternat, T. 7

Teixeira, N. 2, 3, 13

Thirlwell, C.J. 11, 12, 29

'T Jong, G. 8

Trouille, F. 3

Tsang, V. 8

Vadalà, M. 30, 31

Velazquez Ramos, P.M. 32

Yardley, H. 25, 26, 27

Yassin, M. 24

Zaslawski, Z. 9 karger@karger.com

www.karger.com/mca

2020 The Author(s)

Published by S. Karger AG, Basel

Karger 